\title{
A Quantitative Ethnobotanical Study of Plants Used by the Locals in Camiguin Island, Calayan, Cagayan, Philippines
}

\author{
Rudolph Valentino A. Docot ${ }^{1}$, Amritveer Singh Sohal ${ }^{1}$, Carylle G. Dela Cruz ${ }^{1}$, Kaye Allison P. \\ Fontillas ${ }^{1}$, Marc Allan G. Rodriguez ${ }^{1}$, George Emmanuel M. Trillana ${ }^{1}$, Mark Lloyd G. Dapar *2,3 \\ ${ }^{1}$ Department of Biological Sciences, Institute of Art and Sciences, Far Eastern University, Nicanor Reyes Sr. \\ Street, Sampaloc 1015, Manila, Philippines \\ ${ }^{2}$ Department of Biology, College of Arts and Sciences, Central Mindanao University, Musuan, 8714, Bukidnon, \\ Philippines \\ ${ }^{3}$ Microtechnique and Systematics Laboratory, Natural Science Research Center; and Center for Biodiversity \\ Research and Extension in Mindanao, Central Mindanao University, Musuan, 8714, Bukidnon, Philippines
}

Article history:

Submission June 2021

Revised July 2021

Accepted October 2021

*Corresponding author:

E-mail: mackybio.cmu@gmail.com

\begin{abstract}
Traditional knowledge concerning plant utilization in the Philippines is common but is largely underappreciated. Assessment of plant resources by groups of people helps promote valorization of cultural heritage. However, particularly in the Camiguin Island of the North, Cagayan (not to be confused with the island province of Camiguin in Mindanao), the use of terrestrial plants remains unknown. As a response, this study aims to identify island terrestrial plants with ethnobotanical importance through semi-structured interviews. The data collected were then analyzed using quantitative ethnobotanical methods. A total of 70 species belonging to 65 genera and 44 families were surveyed from the 33 informants. The statistical analysis showed a significant difference between male and female informants in ethnobotanical knowledge. Lamiaceae was the most represented plant family with five species recorded. Leaves were the most used plant part (51\%), congruent to previous ethnobotanical studies in the Philippines. Most medicinal preparations were done through decoctions (41.03\%). Much of the medicinal plants surveyed were administered through topical medication (54.90\%), and this is because the occupation of the people of Camiguin island mostly involves heavy physical activities. Thus, the probability of being wounded in the area is very high. It was found that Vitex negundo was the most used plant in Camiguin island with the used value of 0.15 . Furthermore, five ailment/disease categories obtained a $100 \%$ fidelity level. This result indicates that the plants used for that disease category are considered the most preferred species. Respiratory diseases obtained the highest FIC value of 0.40 , indicating species within that category are the best candidates to search for bioactive compounds. This documentation provides the first checklist of useful plants in Camiguin island and will serve as a basis for future ethnobotanical studies in the Philippines.
\end{abstract}

Keywords: Camiguin Island, Ethnobotany, Food, Medicinal plants, Philippines, Quantitative

\section{Introduction}

Valorization of traditional plant knowledge highlights the scope of ethnobotanical studies. The relationships between people and plants embed cultural history $[1,2]$. Plants also affect every aspect of man's existence [3]. Virtually traditional knowledge is passed down orally through generations and is vulnerable to oblivion [4].
The undocumented plant knowledge of indigenous peoples could lead to the loss of traditional practice using the plant community resources. Hence, the preservation of traditional knowledge is relevant. Ethnobotanical research can help preserve the traditional practices and belief systems among indigenous peoples or cultural communi- 
ties in the Philippines and other countries [5].

There are many plant ethnotaxa that are traditionally used in the Philippines. Many native communities use these resources for various nutritional and medicinal applications validated for safety and efficacy [6-11]. Traditional plants use has been proven to be a lucrative source of natural products with myriad biotechnological, pharmaceutical, and other economic and medical applications [12]. The pharmaceutical field has benefited from ethnobotanical studies by selecting the species that has medicinal properties to fill the inadequate pharmacopeia [13]. Proper manage-ment of culturally important medicinal plants must be observed to sustain the income of the locals using the plant community resources [14].

Information is gathered on the importance of indigenous plants and how the herbs and shrubs are cultivated [15]. The great diversity of plants provides food, clothing, shelter, fuel, medicine and was an integral part of the development of modern civilization [16]. Ethnobotanicals are key to drug discovery, but several ethnobotanical plants remain unidentified and unknown [17], particularly in the Philippines [18, 19].

Northern Camiguin Island, not to be confused with the Camiguin province in northern Mindanao, is a volcanic island (c. $166 \mathrm{~km}^{2}$ ) isolated from the rest of Luzon during the typhoon months since it is dangerous to cross because the winds of the island can be hostile and unpredictable (Figure 1). Mount Camiguin de Babuyanes (712 m; Figure 1C), an active stratovolcano that erupted last 1857, is located [20]. Surrounded by the Babuyan Channel, the island is divided into Balatubat, Naguilian, and Minabel, with about 5000 native residents (Figure 1A). The island is home to diverse terrestrial and marine plants [21]. Histo-rical records show 124 bird species from the island group, 56 of which were resident [22]. One threatened species (Yellow Bunting) and four near-threatened species have been recorded, while 13 subspecies of endemic birds to the Babuyan Islands were identified. Fifteen mammal species have been recorded, of which 11 were marine mammals [23, 24]. Few biodiversity studies have been conducted in Camiguin island, focusing on fauna. In addition, the exact number and the way how local people of the island utilize these important natural resources are also unknown. Considering this gap of knowledge, this study aims to document the ethnobotanical uses of terrestrial plant species found on the island of Camiguin de Babuyanes.

\section{Material and Methods \\ Research ethics}

A certificate of approval from the Far Eastern University (FEU) Ethics Committee was obtained to perform interviews with the informants. In addition, prior informed consents (PIC) were also obtained from each of the three barangays of the island. A gratuitous permit (R2-2018-11) issued by the Department of Environment and Natural Resources (DENR) Region II was also obtained to collect plant samples.

\section{Study sites and interview}

The study was conducted within the three Barangays of Camiguin Island, namely Balatubat, Minabel, and Naguilian (Figure 1A). The researchers used random and purposive sampling methods to select the informants, focusing on their traditional healers. 18-39 years old were considered young informants for the age grouping, while 40-84 years old were deemed to be old informants. Each participant was interviewed via semistructured questionnaires in Tagalog and Ilocano, translating into English. The sample questionnaire used in this study followed a template [25] with slight modifications.

\section{Data collection, plant identification, and herbar- ium deposition}

The gathering of ethnobotanical data and plant collection was done a whole year round to observe plant species' phenology and seasonal variations. Each plant species collected during the interview was obtained to prepare herbarium, spirit, and silica-dried leaf materials used for molecular identification. Each sample is folded with news-papers and placed in a polyethylene bag. Dena-tured alcohol was poured into the bag to prevent abscisic acid activity from preserving the plants for processing. Each species' second or third youngest leaf was cut into pieces and placed in zip-lock bags with silica gel for molecular study [26]. Reproductive parts, including the flowers and fruits, were preserved at $70 \%$ ethanol to obtain a well-preserved spirit specimen. The collected plant samples were initially identified through available plant identification tools, references, and platforms like the Co's Digital Flora of the Philippines [27]. Authentication of the collected specimens was done by Mr. Danilo N. Tandang of the Phili- 


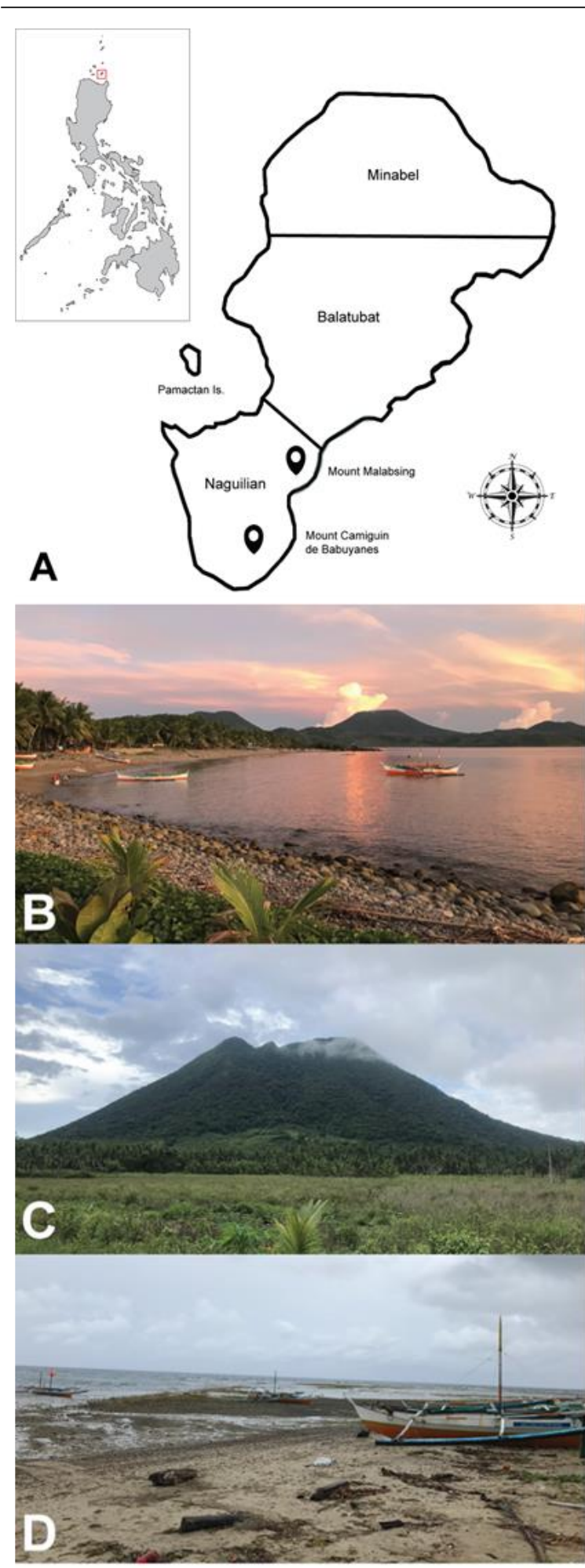

Figure 1. Camiguin Island. A. Map showing the boundaries between the three barangays (inset: location of the island in the Philippines). B. Balatubat port. C. Mount Camiguin de Babuyanes. D. Seashore of Barangay Minabel during low tide. (Photos by R.V.A. Docot). ppine National Herbarium (PNH). All voucher specimens were deposited in the Far Eastern University Herbarium (FEUH) (see table 2 for the accession code of each specimen).

\section{Data and statistical analyses}

Statistical analysis was applied to the study to measure and compare the gathered data from the informants concerning the uses of terrestrial plants. The researchers determined if there is a significant difference between two or more groups such as age, sex, educational attainment, and location. Parametric inferential statistics such as oneway ANOVA using PASW Statistics v.18 [28] was used in this study with the level of significance at 0.05 . This statistical analysis will provide a reasonable supposition about valid information for an entire population when only a subsample of people on the island are surveyed [29].

\section{Plant use categories}

The gathered data through survey interviews was used to classify the plants based on the categories acquired by the researchers. The given information by the informants was used to determine the diseases, symptoms, and their uses.

\section{Use report}

The surveyed and collected plant species was used for a distinctive purpose. If an informant uses the species in more than one purpose under the same category, it is considered as one use-report [30]. When at least two informants used the same plant for similar purposes, a multiple use-report is noted.

\section{Use value (UV)}

The use value (UV) provides a quantitative measure for the comparative importance of a species being used [18]. The value was based on the number of uses and the amount of people that cited a given plant. The value was computed on each species using the following formula: $\mathrm{UV}=(\Sigma$ $\mathrm{Ui} / \mathrm{N}$; Ui was used as the number of use-reports mentioned by each informant for the given species, and $\mathrm{N}$ was used as the total number of informants in the area in which the survey was conducted [18,31-33].

\section{Fidelity level (FL)}

The fidelity level of each ailment/disease cate- 
gory was calculated using the formula: FL $(\%)=$ $(\mathrm{Np} / \mathrm{N}) \times 100$. The $\mathrm{Np}$ in the formula stands for the number of species with a particular use for a specific category, and $\mathrm{N}$ was the total number of use report of the species mentioned in the category. High FL values (near 100\%) indicate that the identified plants have the same uses, while low FL values imply that the named plants were used for different purposes [18].

\section{Informant Consensus Factor (FIC)}

Only diseases were categorized based on the information provided by different informants and was calculated by using the Informants Consensus Factor [FIC $=$ NUR - NT / NUR -1 ]; NUR is the number of use reports in a particular category and NT is the number of taxa or species that are used in each category. FIC value ranges between 0 and 1 , where 1 indicates the highest value [18]. It is important to produce more information about the use of medicinal plants [4]. FIC is measured to study the total usage of plant species according to culture applicability [31-33].

\section{Results and Discussions}

\section{Demographic profile of the informants}

A total of 33 informants were interviewed from the three barangays of the island namely Balatubat, Minabel and Naguillan. The highest number of informants were from Barangay Naguillan (20) whereas the lowest number came from Balatubat (3). Limited key informants were interviewed since most of the people were not in the island and due to bad weather condition during the conduct of interview and fieldwork. The researchers were advised to go back to mainland Luzon which changes the time allotted for the interview or security reasons.

\section{Statistical analysis}

Statistical results of plant knowledge were assessed among the respondents when compared to age, sex, educational attainment, and location of the respondents were tabulated in Table 1.

Age

Results showed no significant difference ( $p=$ 0.672 ) between the age groups in terms of ethnobotanical knowledge. Therefore, the informants age does not affect their ethnobotanical knowledge. Elders (40 years old and above) have a higher mean rank compared to the young age group. Old age group are mostly practicing traditional medicine while the young age group usually stay at home.

\section{Sex}

Results showed that the sex of the respondents has significant difference ( $p=0.003)$ on the ethnobotanical knowledge. Male informants have more plant knowledge compared to females. Females in the island usually stay at home, while males are usually working in the forest to gather food, medicines, and other useful plants for livelihood and living. This is parallel with other ethnic groups in the Philippines that males have more plant knowledge [18, 31].

\section{Educational attainment}

There is no significant difference $(p=0.884)$ on the informant's educational attainment and ethnobotanical knowledge. Results revealed that informants who finished (or at least attended) primary level education have the highest mean rank.

Table 1. Demographic data with mean ranks and test statistics.

\begin{tabular}{|c|c|c|c|c|}
\hline Factors & Variable & No. of Respondents & Mean Rank & Significant Difference \\
\hline \multirow{2}{*}{ Age } & Adolescents (18-39) & 12 & 3.4 & 0.672 \\
\hline & Elders (40 and above) & 21 & 3.8 & \\
\hline \multirow{2}{*}{ Sex } & Male & 15 & 5.2 & 0.003 \\
\hline & Female & 18 & 2.4 & \\
\hline \multirow{3}{*}{$\begin{array}{l}\text { Educational } \\
\text { attainment }\end{array}$} & Primary & 9 & 3.818 & 0.884 \\
\hline & Secondary & 18 & 3.812 & \\
\hline & Tertiary & 6 & 3.2 & \\
\hline \multirow{3}{*}{ Barangay } & Balatubat & 3 & 2.0 & 0.460 \\
\hline & Minabel & 10 & 3.4 & \\
\hline & Naguilian & 20 & 4.1 & \\
\hline
\end{tabular}


They cited more use reports than those who finished (or at least attended) secondary and tertiary level education primarily due to availabi-lity as they usually stay at home and utilize the plants available around them.

\section{Location (Barangays/Villages)}

There is no significant difference ( $p=0.460)$ in the informants' plant knowledge among the three barangays or villages. Therefore, the location of the respondents on the island does not affect their ethnobotanical knowledge. Barangay Naguillan obtained the highest mean rank among the three barangays surveyed since Naguillan has intact vegetation or forests. More plants are available in this village, unlike in the other two barangays with limited vegetation.

\section{Characteristics of the collected plant samples Taxonomy and distribution}

A total of 70 species belonging to 41 families were collected, five of which were not identified up to species level. The collection comprises 35 species native to the Philippines of which three are endemic, whereas the remaining are not native (Table 2). The most represented family is Lamiaceae with five species, followed by Annonaceae, Euphorbiaceae, Myrtaceae, and Zingiberaceae, all represented by four species.

The results are congruent with other ethnobotanical expeditions in the Southern part of Philippine archipelago [18,31-33]. Unlike in other localities, there is a significant difference of knowledge to participants living in an urbanized area with hospital, clinics, and pharmacies, giving the locals the opportunities to access over-the-counter drugs or medicines compared to rural areas [34].

\section{Plant part used, preparation, mode of admin-} istration, and possible side effects

Most of the plants are accustomed to food and treatment of various ailments (Table 2). Based on the present study gathered data, leaves are the most utilized part (51\%), followed by fruit (25\%), stem (11\%), flower (11\%), and roots (1\%). This corresponds to similar results reported in several ethnobotanical studies in Philippines [18,31-36].

The researchers observed that the informants utilize leaves to identify if the plant is used as medicine, insect repellent, food, industrial use, and decoration. Underground organs such as roots were less utilized based on the present study data
(1\%), whereas the aerial organs were vastly used. The use of roots will cause the plant to die, so roots are less utilized to avoid mortality rate increase of plants [36].

The percentage of preparation and administration was computed by determining the number of each category divided by the total number of categories multiplied by 100 . The leading process of preparation is by boiling or decoction (41.03\%), followed by using fresh plants (34.62\%), and crushing (24.36\%). The data regarding plant parts used, preparation, and administration are summarized in Table 2.

The highest percentage of administration was obtained by topical medication (54.90\%) through directly applying the extract on wound) followed by oral $(43.30 \%)$ and inhalation (1.96\%). The informants used both internal and external routes of administration to treat illness. Most people's occupation in Camiguin Island involves heavy physical activities such as fishing and gathering of woods in the forest. These common livelihoods increase the chance of being wounded. Since immediate medication is impossible in the forest, most locals utilize the plants around them as an alternative for wound medication.

Based on the survey among the locals, adverse or side effects of using their medicinal plants were not observed. Instead, the identified plants effectively treat their various health conditions and illness.

\section{Use value}

The highest use-value among the collected 71 species is 0.15 , whereas the lowest is 0.03 . Vitex negundo L. (lagundi) obtained the highest use value with five use reports, followed by Artemisia vulgaris L. (erbaka) and Andrographis paniculata (Burm.f.) Nees (white flower) both with 0.12 usevalue. Plants with high use value are easy to obtain due to their commonality.

The food category obtained the highest number of plant species with 21 reports. The island is often cut from the rest of Luzon during the typhoon months of July to September and during the windy habagat (monsoon) months of October to January [21]. With this, transportation of goods, including food, is difficult, so people use the plants around them as an alternative. This explains the results why most of the plants surveyed were utilized as food. 
Tabel 2. List of medicinal plants, parts used, use category, administration, uses, and use value among the locals of Camiguin Island, Cagayan, Philippines. The language of each common name is informed in parathesis following the abbreviations of [42]. ${ }^{\text {a }}$ Fr- Fruit; Lf- leaf; Fl- Flower; St- Stem; Pe- Petal; Rt- Root

\begin{tabular}{|c|c|c|c|c|c|c|c|c|c|}
\hline No. & Family & Species & $\begin{array}{l}\text { Common } \\
\text { name }\end{array}$ & $\begin{array}{l}\text { Acces- } \\
\text { sion no. } \\
\text { of the } \\
\text { voucher } \\
\text { speci- } \\
\text { men }\end{array}$ & $\begin{array}{l}\text { Distri- } \\
\text { bution } \\
\text { in the } \\
\text { Philip- } \\
\text { pines }\end{array}$ & $\begin{array}{l}\text { Plan } \\
\text { t } \\
\text { part } \\
\text { used } \\
\text { a }\end{array}$ & $\begin{array}{l}\text { Admin- } \\
\text { istration of } \\
\text { prepara- } \\
\text { tion }\end{array}$ & $\begin{array}{l}\text { Diseases } \\
\text { or pur- } \\
\text { pose }\end{array}$ & $\begin{array}{l}\text { Use } \\
\text { value }\end{array}$ \\
\hline 1 & $\begin{array}{l}\text { Acan- } \\
\text { thaceae }\end{array}$ & $\begin{array}{l}\text { Justicia } \\
\text { gendarussa } \\
\text { Burm.f. }\end{array}$ & $\begin{array}{l}\text { puli puli } \\
\text { (Ilk.) }\end{array}$ & $\begin{array}{l}\text { FEUH } \\
003135\end{array}$ & Native & Lf & $\begin{array}{l}\text { Drink de- } \\
\text { coction }\end{array}$ & $\begin{array}{l}\text { Kidney } \\
\text { stones }\end{array}$ & 0.03 \\
\hline 2 & $\begin{array}{l}\text { Acan- } \\
\text { thaceae }\end{array}$ & $\begin{array}{l}\text { Androgra- } \\
\text { phis panic- } \\
\text { ulata } \\
\text { (Burm.f.) } \\
\text { Nees. }\end{array}$ & $\begin{array}{l}\text { white } \\
\text { flower } \\
\text { (Eng.) }\end{array}$ & $\begin{array}{l}\text { FEUH } \\
003164\end{array}$ & $\begin{array}{l}\text { Not na- } \\
\text { tive }\end{array}$ & Lf & $\begin{array}{l}\text { Drink de- } \\
\text { coction }\end{array}$ & $\begin{array}{l}\text { Stomach- } \\
\text { ache, } \\
\text { Abortion } \\
\text { and Ma- } \\
\text { laria }\end{array}$ & 0.12 \\
\hline 3 & $\begin{array}{l}\text { Amaran- } \\
\text { thaceae }\end{array}$ & $\begin{array}{l}\text { Achy- } \\
\text { ranthes } \\
\text { aspera L. }\end{array}$ & garem (Ilk.) & $\begin{array}{l}\text { FEUH } \\
003229\end{array}$ & $\begin{array}{l}\text { Not na- } \\
\text { tive }\end{array}$ & Lf & $\begin{array}{l}\text { Apply ex- } \\
\text { tract on the } \\
\text { wound }\end{array}$ & Wounds & 0.03 \\
\hline 4 & $\begin{array}{l}\text { Amarylli- } \\
\text { daceae }\end{array}$ & $\begin{array}{l}\text { Allium } \\
\text { tuberosum } \\
\text { Rottler ex } \\
\text { Spreng. }\end{array}$ & $\begin{array}{l}\text { kutsay } \\
\text { (ManyLgs.) }\end{array}$ & $\begin{array}{l}\text { FEUH } \\
003154\end{array}$ & $\begin{array}{l}\text { Not na- } \\
\text { tive }\end{array}$ & Lf & $\begin{array}{l}\text { Apply ex- } \\
\text { tract on } \\
\text { wound; } \\
\text { Used as in- } \\
\text { gredients } \\
\text { for cook- } \\
\text { ing }\end{array}$ & Wounds & 0.09 \\
\hline 5 & $\begin{array}{l}\text { An- } \\
\text { nonaceae }\end{array}$ & $\begin{array}{l}\text { Annona } \\
\text { muricata } \\
\text { L. }\end{array}$ & $\begin{array}{l}\text { guyabano } \\
\text { (ManyLgs.) }\end{array}$ & $\begin{array}{l}\text { FEUH } \\
003137\end{array}$ & $\begin{array}{l}\text { Not na- } \\
\text { tive }\end{array}$ & Lf & $\begin{array}{l}\text { Drink de- } \\
\text { coction }\end{array}$ & $\begin{array}{l}\text { Kidney } \\
\text { stones }\end{array}$ & 0.09 \\
\hline 6 & $\begin{array}{l}\text { An- } \\
\text { nonaceae }\end{array}$ & $\begin{array}{l}\text { Annona } \\
\text { squamosa } \\
\text { L. }\end{array}$ & $\begin{array}{l}\text { atis } \\
\text { (ManyLgs.) } \\
\text {; dalayap } \\
\text { (Ilk.) }\end{array}$ & $\begin{array}{l}\text { FEUH } \\
003142\end{array}$ & $\begin{array}{l}\text { Not na- } \\
\text { tive }\end{array}$ & Lf & $\begin{array}{l}\text { Drink de- } \\
\text { coction }\end{array}$ & $\begin{array}{l}\text { Fever } \\
\text { and uri- } \\
\text { nary tract } \\
\text { infec- } \\
\text { tion }\end{array}$ & 0.03 \\
\hline 7 & $\begin{array}{l}\text { An- } \\
\text { nonaceae }\end{array}$ & Uvaria sp. & None & $\begin{array}{l}\text { FEUH } \\
003131\end{array}$ & $\mathrm{n} / \mathrm{a}$ & Fr & $\begin{array}{l}\text { Eat } \\
\text { chewed }\end{array}$ & Food & 0.03 \\
\hline 8 & Apiaceae & $\begin{array}{l}\text { Centella } \\
\text { asiatica } \\
\text { (L.) Urb. }\end{array}$ & $\begin{array}{l}\text { marakobibi } \\
\text { (Ilk.) }\end{array}$ & $\begin{array}{l}\text { FEUH } \\
003177\end{array}$ & Native & Lf & $\begin{array}{l}\text { Drink de- } \\
\text { coction }\end{array}$ & Cough & 0.03 \\
\hline 9 & $\begin{array}{l}\text { Apocyna- } \\
\text { ceae }\end{array}$ & $\begin{array}{l}\text { Plumeria } \\
\text { obtusa L. }\end{array}$ & $\begin{array}{l}\text { calachuchi } \\
\text { (Eng.) }\end{array}$ & $\begin{array}{l}\text { FEUH } \\
003143\end{array}$ & $\begin{array}{l}\text { Not na- } \\
\text { tive }\end{array}$ & $\begin{array}{l}\text { Lf, } \\
\text { Fl }\end{array}$ & $\begin{array}{l}\text { Apply the } \\
\text { extracts to } \\
\text { affected } \\
\text { parts }\end{array}$ & Acne & 0.06 \\
\hline 10 & $\begin{array}{l}\text { Apocyna- } \\
\text { ceae }\end{array}$ & $\begin{array}{l}\text { Tabernae- } \\
\text { montana } \\
\text { pan- } \\
\text { dacaqui } \\
\text { Poir. }\end{array}$ & $\begin{array}{l}\text { busbusilak } \\
\text { (Ilk.) }\end{array}$ & $\begin{array}{l}\text { FEUH } \\
003778\end{array}$ & Native & Lf & $\begin{array}{l}\text { Apply ex- } \\
\text { tracts on } \\
\text { wound }\end{array}$ & Wounds & 0.03 \\
\hline 11 & $\begin{array}{l}\text { Apocyna- } \\
\text { ceae }\end{array}$ & $\begin{array}{l}\text { Alstonia } \\
\text { scholaris } \\
\text { (L.) R.Br. }\end{array}$ & $\begin{array}{l}\text { dalipawan } \\
\text { (Ilk.) }\end{array}$ & $\begin{array}{l}\text { FEUH } \\
003779\end{array}$ & Native & $\mathrm{Fr}$ & $\begin{array}{l}\text { Eat } \\
\text { chewed }\end{array}$ & Food & 0.09 \\
\hline 12 & Arecaceae & $\begin{array}{l}\text { Calamus } \\
\text { sp. }\end{array}$ & $\begin{array}{l}\text { alanyog } \\
\text { (Ilk.) }\end{array}$ & $\begin{array}{l}\text { FEUH } \\
003207\end{array}$ & $\mathrm{n} / \mathrm{a}$ & $\mathrm{Fr}$ & $\begin{array}{l}\text { Eat } \\
\text { chewed }\end{array}$ & Food & 0.03 \\
\hline 13 & Arecaceae & $\begin{array}{l}\text { Pinanga } \\
\text { insignis } \\
\text { Becc. }\end{array}$ & $\begin{array}{l}\text { rasidan } \\
\text { (Ilk.) }\end{array}$ & $\begin{array}{l}\text { FEUH } \\
003124\end{array}$ & Native & Fr & $\begin{array}{l}\text { Eat } \\
\text { chewed }\end{array}$ & Food & 0.03 \\
\hline
\end{tabular}




\begin{tabular}{|c|c|c|c|c|c|c|c|c|c|}
\hline No. & Family & Species & $\begin{array}{l}\text { Common } \\
\text { name }\end{array}$ & $\begin{array}{l}\text { Acces- } \\
\text { sion no. } \\
\text { of the } \\
\text { voucher } \\
\text { speci- } \\
\text { men }\end{array}$ & $\begin{array}{l}\text { Distri- } \\
\text { bution } \\
\text { in the } \\
\text { Philip- } \\
\text { pines }\end{array}$ & $\begin{array}{l}\text { Plan } \\
\mathrm{t} \\
\text { part } \\
\text { used } \\
\mathrm{a}\end{array}$ & $\begin{array}{l}\text { Admin- } \\
\text { istration of } \\
\text { prepara- } \\
\text { tion }\end{array}$ & $\begin{array}{l}\text { Diseases } \\
\text { or pur- } \\
\text { pose }\end{array}$ & $\begin{array}{l}\text { Use } \\
\text { value }\end{array}$ \\
\hline 14 & $\begin{array}{l}\text { Aspleni- } \\
\text { aceae }\end{array}$ & $\begin{array}{l}\text { Asplenium } \\
\text { nidus L. }\end{array}$ & $\begin{array}{l}\text { kapa kapa } \\
\text { (Ilk.) }\end{array}$ & $\begin{array}{l}\text { FEUH } \\
003121\end{array}$ & Native & Lf & $\begin{array}{l}\text { Apply the } \\
\text { extracts to } \\
\text { affected } \\
\text { parts }\end{array}$ & Edema & 0.03 \\
\hline 15 & Asteraceae & $\begin{array}{l}\text { Artemisia } \\
\text { vulgaris L. }\end{array}$ & $\begin{array}{l}\text { erbaka } \\
\text { (Ilk.) }\end{array}$ & $\begin{array}{l}\text { FEUH } \\
003134\end{array}$ & $\begin{array}{l}\text { Not na- } \\
\text { tive }\end{array}$ & Lf & $\begin{array}{l}\text { Apply di- } \\
\text { rectly on } \\
\text { the wound; } \\
\text { Drink de- } \\
\text { coction }\end{array}$ & $\begin{array}{l}\text { Cough } \\
\text { and Fe- } \\
\text { ver }\end{array}$ & 0.12 \\
\hline 16 & Bixaceae & $\begin{array}{l}\text { Bixa orel- } \\
\text { lana L. }\end{array}$ & asuete (Ilk.) & $\begin{array}{l}\text { FEUH } \\
003130\end{array}$ & $\begin{array}{l}\text { Not na- } \\
\text { tive }\end{array}$ & Lf & - & $\begin{array}{l}\text { Handi- } \\
\text { craft }\end{array}$ & 0.03 \\
\hline 17 & Cactaceae & $\begin{array}{l}\text { Opuntia } \\
\text { stricta } \\
\text { (Haw.) } \\
\text { Haw. }\end{array}$ & $\begin{array}{l}\text { Aloe vera } \\
\text { (Eng.) }\end{array}$ & $\begin{array}{l}\text { FEUH } \\
003156\end{array}$ & $\begin{array}{l}\text { Not na- } \\
\text { tive }\end{array}$ & $\mathrm{Pe}$ & $\begin{array}{l}\text { Apply the } \\
\text { extracts to } \\
\text { affected } \\
\text { parts }\end{array}$ & Hair fall & 0.03 \\
\hline 18 & $\begin{array}{l}\text { Canna- } \\
\text { baceae }\end{array}$ & $\begin{array}{l}\text { Celtis } \\
\text { philippen- } \\
\text { sis Blanco }\end{array}$ & uratan (Ilk.) & $\begin{array}{l}\text { FEUH } \\
003162\end{array}$ & Native & $\mathrm{Fr}$ & $\begin{array}{l}\text { Eat } \\
\text { chewed }\end{array}$ & Food & 0.03 \\
\hline 19 & Caricaceae & $\begin{array}{l}\text { Carica pa- } \\
\text { paya L. }\end{array}$ & $\begin{array}{l}\text { papaya } \\
\text { (ManyLgs.) }\end{array}$ & $\begin{array}{l}\text { FEUH } \\
003780\end{array}$ & $\begin{array}{l}\text { Not na- } \\
\text { tive }\end{array}$ & $\begin{array}{l}\text { Lf, } \\
\text { Fr }\end{array}$ & $\begin{array}{l}\text { Apply ex- } \\
\text { tracts on } \\
\text { wound; } \\
\text { Eat } \\
\text { chewed; } \\
\text { Drink de- } \\
\text { coction }\end{array}$ & $\begin{array}{l}\text { Appendi- } \\
\text { citis and } \\
\text { wounds }\end{array}$ & 0.09 \\
\hline 20 & $\begin{array}{l}\text { Combreta- } \\
\text { ceae }\end{array}$ & $\begin{array}{l}\text { Terminalia } \\
\text { catappa L. }\end{array}$ & $\begin{array}{l}\text { talisay } \\
\text { (ManyLgs.) }\end{array}$ & $\begin{array}{l}\text { FEUH } \\
003161\end{array}$ & Native & $\mathrm{Fr}$ & $\begin{array}{l}\text { Eat } \\
\text { chewed }\end{array}$ & Food & 0.03 \\
\hline 21 & $\begin{array}{l}\text { Comme- } \\
\text { linaceae }\end{array}$ & $\begin{array}{l}\text { Tradescan- } \\
\text { tia } \\
\text { spathacea } \\
\text { Sw. }\end{array}$ & $\begin{array}{l}\text { uki uki } \\
\text { (Ilk.) }\end{array}$ & $\begin{array}{l}\text { FEUH } \\
003175\end{array}$ & $\begin{array}{l}\text { Not na- } \\
\text { tive }\end{array}$ & Lf & $\begin{array}{l}\text { Drink de- } \\
\text { coction }\end{array}$ & $\begin{array}{l}\text { Menstru- } \\
\text { ation }\end{array}$ & 0.03 \\
\hline 22 & $\begin{array}{l}\text { Composi- } \\
\text { tae }\end{array}$ & $\begin{array}{l}\text { Blumea } \\
\text { balsamif- } \\
\text { era (L.) } \\
\text { DC. }\end{array}$ & $\begin{array}{l}\text { subusob } \\
\text { (Ibl, Ilk.) }\end{array}$ & $\begin{array}{l}\text { FEUH } \\
003179\end{array}$ & Native & Lf & $\begin{array}{l}\text { Use for } \\
\text { bathing }\end{array}$ & Hygiene & 0.03 \\
\hline 23 & $\begin{array}{l}\text { Composi- } \\
\text { tae }\end{array}$ & $\begin{array}{l}\text { Mikania } \\
\text { cordata } \\
\text { (Burm.f.) } \\
\text { B.L.Rob }\end{array}$ & $\begin{array}{l}\text { kumot ku- } \\
\text { mot (Ilk.) }\end{array}$ & $\begin{array}{l}\text { FEUH } \\
003180\end{array}$ & $\begin{array}{l}\text { Not na- } \\
\text { tive }\end{array}$ & Lf & $\begin{array}{l}\text { Apply di- } \\
\text { rectly on } \\
\text { the wound }\end{array}$ & Wounds & 0.03 \\
\hline 24 & $\begin{array}{l}\text { Cucurbita- } \\
\text { ceae }\end{array}$ & $\begin{array}{l}\text { Momor- } \\
\text { dica char- } \\
\text { antia L. }\end{array}$ & $\begin{array}{l}\text { ampalaya } \\
\text { (Tag.) }\end{array}$ & $\begin{array}{l}\text { FEUH } \\
003152\end{array}$ & Native & Lf & $\begin{array}{l}\text { Drink de- } \\
\text { coction }\end{array}$ & Diabetes & 0.06 \\
\hline 25 & $\begin{array}{l}\text { Dilleni- } \\
\text { aceae }\end{array}$ & $\begin{array}{l}\text { Dillenia } \\
\text { philip- } \\
\text { pinensis } \\
\text { Rolfe var. } \\
\text { philip- } \\
\text { pinensis }\end{array}$ & palale (Ilk.) & $\begin{array}{l}\text { FEUH } \\
003173\end{array}$ & $\begin{array}{l}\text { Native } \\
\text { and en- } \\
\text { demic }\end{array}$ & $\mathrm{Fr}$ & $\begin{array}{l}\text { Eat } \\
\text { chewed }\end{array}$ & Food & 0.06 \\
\hline 26 & $\begin{array}{l}\text { Dilleni- } \\
\text { aceae }\end{array}$ & Dillenia sp. & $\begin{array}{l}\text { dalandan } \\
\text { (ManyLgs.) }\end{array}$ & $\begin{array}{l}\text { FEUH } \\
003174\end{array}$ & $\mathrm{n} / \mathrm{a}$ & Lf & $\begin{array}{l}\text { Use for } \\
\text { bathing }\end{array}$ & $\begin{array}{l}\text { Bathing } \\
\text { for new }\end{array}$ & 0.06 \\
\hline
\end{tabular}

Continue... 
RVA Docot, AS Sohal, CGD Cruz et al., 2022 / Ethnobotanical Study of Plants Among the Locals in Camiguin Island, Philippines

\begin{tabular}{|c|c|c|c|c|c|c|c|c|c|}
\hline No. & Family & Species & $\begin{array}{l}\text { Common } \\
\text { name }\end{array}$ & $\begin{array}{l}\text { Acces- } \\
\text { sion no. } \\
\text { of the } \\
\text { voucher } \\
\text { speci- } \\
\text { men }\end{array}$ & $\begin{array}{l}\text { Distri- } \\
\text { bution } \\
\text { in the } \\
\text { Philip- } \\
\text { pines }\end{array}$ & $\begin{array}{l}\text { Plan } \\
t \\
\text { part } \\
\text { used } \\
\text { a }\end{array}$ & $\begin{array}{l}\text { Admin- } \\
\text { istration of } \\
\text { prepara- } \\
\text { tion }\end{array}$ & $\begin{array}{c}\text { Diseases } \\
\text { or pur- } \\
\text { pose }\end{array}$ & $\begin{array}{c}\text { Use } \\
\text { value }\end{array}$ \\
\hline 27 & Ebenaceae & $\begin{array}{l}\text { Diospyros } \\
\text { lanceifolia } \\
\text { Roxb. }\end{array}$ & $\begin{array}{l}\text { balinagta } \\
\text { (Ilk.) }\end{array}$ & $\begin{array}{l}\text { FEUH } \\
003128\end{array}$ & Native & Fr & $\begin{array}{l}\text { Eat } \\
\text { chewed }\end{array}$ & $\begin{array}{l}\text { born } \\
\text { child } \\
\text { Food }\end{array}$ & 0.03 \\
\hline 28 & $\begin{array}{l}\text { Euphorbia- } \\
\text { ceae }\end{array}$ & $\begin{array}{l}\text { Antidesma } \\
\text { montanum } \\
\text { Blume var. } \\
\text { montanum }\end{array}$ & $\begin{array}{l}\text { bugnay } \\
\text { (Ibg, Ilk.) }\end{array}$ & $\begin{array}{l}\text { FEUH } \\
003139\end{array}$ & Native & Lf & $\begin{array}{l}\text { Drink de- } \\
\text { coction }\end{array}$ & Fever & 0.09 \\
\hline 29 & $\begin{array}{l}\text { Euphorbia- } \\
\text { ceae }\end{array}$ & $\begin{array}{l}\text { Euphorbia } \\
\text { hirta L. }\end{array}$ & $\begin{array}{l}\text { maragatas } \\
\text { (Ilk.) }\end{array}$ & $\begin{array}{l}\text { FEUH } \\
003171\end{array}$ & $\begin{array}{l}\text { Not na- } \\
\text { tive }\end{array}$ & St & $\begin{array}{l}\text { Apply the } \\
\text { extracts to } \\
\text { affected } \\
\text { parts }\end{array}$ & Sore eyes & 0.03 \\
\hline 30 & $\begin{array}{l}\text { Euphorbia- } \\
\text { ceae }\end{array}$ & $\begin{array}{l}\text { Jatropha } \\
\text { curcas L. }\end{array}$ & $\begin{array}{l}\text { tawa tawa } \\
\text { (Ilk.) }\end{array}$ & $\begin{array}{l}\text { FEUH } \\
003153\end{array}$ & $\begin{array}{l}\text { Not na- } \\
\text { tive }\end{array}$ & Lf & $\begin{array}{l}\text { Drink de- } \\
\text { coction }\end{array}$ & Dengue & 0.03 \\
\hline 31 & $\begin{array}{l}\text { Euphorbia- } \\
\text { ceae }\end{array}$ & $\begin{array}{l}\text { Hevea } \\
\text { brasiliensis } \\
\text { (Willd. ex } \\
\text { A.Juss.) } \\
\text { Müll. Arg. }\end{array}$ & $\begin{array}{l}\text { kuniber } \\
\text { (Ilk.) }\end{array}$ & $\begin{array}{l}\text { FEUH } \\
003184\end{array}$ & $\begin{array}{l}\text { Not na- } \\
\text { tive }\end{array}$ & Lf & $\begin{array}{l}\text { Apply di- } \\
\text { rectly on } \\
\text { the wound }\end{array}$ & Wounds & 0.03 \\
\hline 32 & Fabaceae & $\begin{array}{l}\text { Senna } \\
\text { alata (L.) } \\
\text { Roxb. }\end{array}$ & $\begin{array}{l}\text { andadasin } \\
\text { (Ilk.) }\end{array}$ & $\begin{array}{l}\text { FEUH } \\
003168\end{array}$ & $\begin{array}{l}\text { Not na- } \\
\text { tive }\end{array}$ & Lf & $\begin{array}{l}\text { Apply } \\
\text { young } \\
\text { leaves on a } \\
\text { forehead }\end{array}$ & Fever & 0.03 \\
\hline 33 & Fabaceae & $\begin{array}{l}\text { Pterocar- } \\
\text { pus indicus } \\
\text { Willd. }\end{array}$ & $\begin{array}{l}\text { dun- } \\
\text { gon/narra }\end{array}$ & $\begin{array}{l}\text { FEUH } \\
003179\end{array}$ & Native & Lf & $\begin{array}{l}\text { Apply } \\
\text { young } \\
\text { leaves on a } \\
\text { forehead }\end{array}$ & $\begin{array}{l}\text { Head } \\
\text { Ache }\end{array}$ & 0.03 \\
\hline 34 & $\begin{array}{l}\text { Hydran- } \\
\text { geaceae }\end{array}$ & $\begin{array}{l}\text { Hydrangea } \\
\text { macro- } \\
\text { phylla } \\
\text { (Thunb.) } \\
\text { Ser. }\end{array}$ & $\begin{array}{l}\text { million } \\
\text { flowers } \\
\text { (Eng.) }\end{array}$ & $\begin{array}{l}\text { FEUH } \\
003182\end{array}$ & $\begin{array}{l}\text { Not na- } \\
\text { tive }\end{array}$ & $\mathrm{Fl}$ & $\begin{array}{l}\text { Display } \\
\text { around a } \\
\text { house or } \\
\text { building } \\
\text { for orna- } \\
\text { mentation }\end{array}$ & $\begin{array}{c}\text { Orna- } \\
\text { mental }\end{array}$ & 0.03 \\
\hline 35 & Lamiaceae & $\begin{array}{l}\text { Ocimum } \\
\text { basilicum } \\
\text { L. }\end{array}$ & tawas (Ilk.) & $\begin{array}{l}\text { FEUH } \\
003132\end{array}$ & Native & Lf & $\begin{array}{l}\text { Apply di- } \\
\text { rectly on } \\
\text { the af- } \\
\text { fected } \\
\text { body parts }\end{array}$ & Sprain & 0.03 \\
\hline 36 & Lamiaceae & $\begin{array}{l}\text { Premna } \\
\text { odorata } \\
\text { Blanco }\end{array}$ & po-on (Ilk.) & $\begin{array}{l}\text { FEUH } \\
003133\end{array}$ & Native & Lf & $\begin{array}{l}\text { Apply the } \\
\text { extracts to } \\
\text { affected } \\
\text { parts }\end{array}$ & Sprain & 0.03 \\
\hline 37 & Lamiaceae & $\begin{array}{l}\text { Vitex } \\
\text { negundo L. }\end{array}$ & $\begin{array}{l}\text { lagundi } \\
\text { (Bik, } \\
\text { BisPn., } \\
\text { Ibg., Tag.) }\end{array}$ & $\begin{array}{l}\text { FEUH } \\
003136\end{array}$ & $\begin{array}{l}\text { Not na- } \\
\text { tive }\end{array}$ & Lf & $\begin{array}{l}\text { Drink de- } \\
\text { coction }\end{array}$ & $\begin{array}{l}\text { Malaria, } \\
\text { Colds, } \\
\text { Cough, } \\
\text { Fever } \\
\text { and } \\
\text { Wounds }\end{array}$ & 0.15 \\
\hline
\end{tabular}

Continue... 


\begin{tabular}{|c|c|c|c|c|c|c|c|c|c|}
\hline No. & Family & Species & $\begin{array}{l}\text { Common } \\
\text { name }\end{array}$ & $\begin{array}{l}\text { Acces- } \\
\text { sion no. } \\
\text { of the } \\
\text { voucher } \\
\text { speci- } \\
\text { men }\end{array}$ & $\begin{array}{l}\text { Distri- } \\
\text { bution } \\
\text { in the } \\
\text { Philip- } \\
\text { pines }\end{array}$ & $\begin{array}{l}\text { Plan } \\
\mathrm{t} \\
\text { part } \\
\text { used } \\
\text { a }\end{array}$ & $\begin{array}{l}\text { Admin- } \\
\text { istration of } \\
\text { prepara- } \\
\text { tion }\end{array}$ & $\begin{array}{l}\text { Diseases } \\
\text { or pur- } \\
\text { pose }\end{array}$ & $\begin{array}{l}\text { Use } \\
\text { value }\end{array}$ \\
\hline 38 & Lamiaceae & $\begin{array}{l}\text { Coleus am- } \\
\text { boinicus } \\
\text { Lour. }\end{array}$ & $\begin{array}{l}\text { oregano } \\
\text { (Ilk, Sp, } \\
\text { Tag) }\end{array}$ & $\begin{array}{l}\text { FEUH } \\
003150\end{array}$ & $\begin{array}{l}\text { Not na- } \\
\text { tive }\end{array}$ & Lf & $\begin{array}{l}\text { Drink de- } \\
\text { coction; } \\
\text { Used as in- } \\
\text { gredients } \\
\text { for cook- } \\
\text { ing }\end{array}$ & $\begin{array}{l}\text { Cough } \\
\text { and } \\
\text { Cooking }\end{array}$ & 0.09 \\
\hline 39 & Lamiaceae & $\begin{array}{l}\text { Coleus } \\
\text { scutellari- } \\
\text { oides (L.) } \\
\text { Benth.. }\end{array}$ & $\begin{array}{l}\text { tamuras } \\
\text { (Ilk.) }\end{array}$ & $\begin{array}{l}\text { FEUH } \\
003160\end{array}$ & Native & $\mathrm{Fr}$ & $\begin{array}{l}\text { Eat } \\
\text { chewed }\end{array}$ & Food & 0.03 \\
\hline 40 & $\begin{array}{l}\text { Legumi- } \\
\text { nosae }\end{array}$ & $\begin{array}{l}\text { Flemingia } \\
\text { javanica } \\
\text { C.Y.Wu }\end{array}$ & $\begin{array}{l}\text { garantin- } \\
\text { wan (Ilk.) }\end{array}$ & $\begin{array}{l}\text { FEUH } \\
003145\end{array}$ & $\begin{array}{l}\text { Not na- } \\
\text { tive }\end{array}$ & Lf & $\begin{array}{l}\text { Apply di- } \\
\text { rectly on } \\
\text { the wound }\end{array}$ & Wounds & 0.03 \\
\hline 41 & $\begin{array}{l}\text { Legumi- } \\
\text { nosae }\end{array}$ & $\begin{array}{l}\text { Antidesma } \\
\text { bunius (L.) } \\
\text { Spreng. }\end{array}$ & $\begin{array}{l}\text { barakbak } \\
\text { (Ilk.) }\end{array}$ & $\begin{array}{l}\text { FEUH } \\
003157\end{array}$ & Native & $\mathrm{Fr}$ & $\begin{array}{l}\text { Eat } \\
\text { chewed }\end{array}$ & Food & 0.03 \\
\hline 42 & Lythraceae & $\begin{array}{l}\text { Lagerstro- } \\
\text { emia speci- } \\
\text { osa (L.) } \\
\text { Pers. }\end{array}$ & $\begin{array}{l}\text { banaba } \\
\text { (ManyLgs.) }\end{array}$ & $\begin{array}{l}\text { FEUH } \\
003230\end{array}$ & Native & Lf & $\begin{array}{l}\text { Apply the } \\
\text { extracts to } \\
\text { affected } \\
\text { parts }\end{array}$ & Edema & 0.06 \\
\hline 43 & Malvaceae & $\begin{array}{l}\text { Hibiscus } \\
\text { rosa-sinen- } \\
\text { sis L. }\end{array}$ & $\begin{array}{l}\text { gumamela } \\
\text { (ManyLgs.) }\end{array}$ & $\begin{array}{l}\text { FEUH } \\
003146\end{array}$ & $\begin{array}{l}\text { Not na- } \\
\text { tive }\end{array}$ & Lf & $\begin{array}{l}\text { Apply the } \\
\text { pounded } \\
\text { leaves to } \\
\text { the af- } \\
\text { fected area }\end{array}$ & Boils & 0.03 \\
\hline 44 & $\begin{array}{l}\text { Maran- } \\
\text { taceae }\end{array}$ & $\begin{array}{l}\text { Donax } \\
\text { canni- } \\
\text { formis } \\
\text { (G.Forst.) } \\
\text { K.Schum. }\end{array}$ & $\begin{array}{l}\text { banban } \\
\text { (ManyLgs.) }\end{array}$ & $\begin{array}{l}\text { FEUH } \\
003126\end{array}$ & Native & $\mathrm{Fr}$ & $\begin{array}{l}\text { Eat } \\
\text { chewed }\end{array}$ & Food & 0.03 \\
\hline 45 & $\begin{array}{l}\text { Menisper- } \\
\text { maceae }\end{array}$ & $\begin{array}{l}\text { Cissampe- } \\
\text { los pareira } \\
\text { L. }\end{array}$ & $\begin{array}{l}\text { laplapsu } \\
\text { (Ilk.) }\end{array}$ & $\begin{array}{l}\text { FEUH } \\
003183\end{array}$ & Native & Lf & $\begin{array}{l}\text { Drink de- } \\
\text { coction }\end{array}$ & Edema & 0.06 \\
\hline 46 & Moraceae & $\begin{array}{l}\text { Artocarpus } \\
\text { altilis (Par- } \\
\text { kinson) } \\
\text { Fosberg }\end{array}$ & $\begin{array}{l}\text { pakak (Ilk, } \\
\text { Ifg.) }\end{array}$ & $\begin{array}{l}\text { FEUH } \\
003122\end{array}$ & Native & $\mathrm{Fr}$ & $\begin{array}{l}\text { Used as in- } \\
\text { gredients } \\
\text { for cook- } \\
\text { ing }\end{array}$ & Diabetes & 0.03 \\
\hline 47 & Myrtaceae & $\begin{array}{l}\text { Rhodomyr- } \\
\text { tus tomen- } \\
\text { tosa (Ai- } \\
\text { ton) Hassk } \\
\text { var. tomen- } \\
\text { tosa }\end{array}$ & $\begin{array}{l}\text { tungaw- } \\
\text { tungaw } \\
\text { (Ilk.) }\end{array}$ & $\begin{array}{l}\text { FEUH } \\
003127\end{array}$ & Native & $\mathrm{Fr}$ & $\begin{array}{l}\text { Eat } \\
\text { chewed }\end{array}$ & Food & 0.03 \\
\hline 48 & Myrtaceae & $\begin{array}{l}\text { Syzygium } \\
\text { fenicis } \\
\text { (C.B.Rob) } \\
\text { Merr. }\end{array}$ & $\begin{array}{l}\text { barakbak } \\
\text { (Ilk.) }\end{array}$ & $\begin{array}{l}\text { FEUH } \\
003781\end{array}$ & $\begin{array}{l}\text { Native } \\
\text { and en- } \\
\text { demic }\end{array}$ & Lf & $\begin{array}{l}\text { Eat } \\
\text { chewed }\end{array}$ & Food & 0.06 \\
\hline 49 & Myrtaceae & $\begin{array}{l}\text { Psidium } \\
\text { guajava L. }\end{array}$ & $\begin{array}{l}\text { bayabas } \\
\text { (BisC., } \\
\text { Ibg., Ilk, } \\
\text { Tag.) }\end{array}$ & $\begin{array}{l}\text { FEUH } \\
003151\end{array}$ & $\begin{array}{l}\text { Not na- } \\
\text { tive }\end{array}$ & $\begin{array}{l}\text { Lf, } \\
\text { Fr }\end{array}$ & $\begin{array}{l}\text { Drink de- } \\
\text { coction; } \\
\text { Apply the } \\
\text { extracts to }\end{array}$ & Cancer & 0.03 \\
\hline
\end{tabular}


RVA Docot, AS Sohal, CGD Cruz et al., 2022 / Ethnobotanical Study of Plants Among the Locals in Camiguin Island, Philippines

\begin{tabular}{|c|c|c|c|c|c|c|c|c|c|}
\hline No. & Family & Species & $\begin{array}{l}\text { Common } \\
\text { name }\end{array}$ & $\begin{array}{l}\text { Acces- } \\
\text { sion no. } \\
\text { of the } \\
\text { voucher } \\
\text { speci- } \\
\text { men }\end{array}$ & $\begin{array}{l}\text { Distri- } \\
\text { bution } \\
\text { in the } \\
\text { Philip- } \\
\text { pines }\end{array}$ & $\begin{array}{l}\text { Plan } \\
t \\
\text { part } \\
\text { used } \\
\text { a }\end{array}$ & $\begin{array}{l}\text { Admin- } \\
\text { istration of } \\
\text { prepara- } \\
\text { tion }\end{array}$ & $\begin{array}{l}\text { Diseases } \\
\text { or pur- } \\
\text { pose }\end{array}$ & $\begin{array}{c}\text { Use } \\
\text { value }\end{array}$ \\
\hline 50 & Myrtaceae & $\begin{array}{l}\text { Syzygium } \\
\text { subrotun- } \\
\text { difolium } \\
\text { (C.B.Rob.) } \\
\text { Merr. }\end{array}$ & $\begin{array}{l}\text { tampuy } \\
\text { (Ilk.) }\end{array}$ & $\begin{array}{l}\text { FEUH } \\
003158\end{array}$ & $\begin{array}{l}\text { Native } \\
\text { and en- } \\
\text { demic }\end{array}$ & Fr & $\begin{array}{l}\text { affected } \\
\text { parts } \\
\text { Eat } \\
\text { chewed }\end{array}$ & Food & 0.03 \\
\hline 51 & $\begin{array}{l}\text { Pan- } \\
\text { danaceae }\end{array}$ & $\begin{array}{l}\text { Pandanus } \\
\text { odorifer } \\
\text { (Forssk.) } \\
\text { Kuntze }\end{array}$ & budak (Ilk.) & $\begin{array}{l}\text { FEUH } \\
003228\end{array}$ & Native & $\begin{array}{l}\text { Lf, } \\
\text { St }\end{array}$ & - & $\begin{array}{l}\text { Handi- } \\
\text { craft }\end{array}$ & 0.03 \\
\hline 52 & $\begin{array}{l}\text { Pan- } \\
\text { danaceae }\end{array}$ & $\begin{array}{l}\text { Pandanus } \\
\text { sp. }\end{array}$ & $\begin{array}{l}\text { sarakat } \\
\text { (Ilk.) }\end{array}$ & $\begin{array}{l}\text { FEUH } \\
003167\end{array}$ & $\mathrm{n} / \mathrm{a}$ & Lf & - & $\begin{array}{l}\text { Handi- } \\
\text { craft }\end{array}$ & 0.03 \\
\hline 53 & $\begin{array}{l}\text { Pan- } \\
\text { danaceae }\end{array}$ & $\begin{array}{l}\text { Pandanus } \\
\text { amarylli- } \\
\text { folius } \\
\text { Roxb. ex } \\
\text { Lindl. }\end{array}$ & $\begin{array}{l}\text { pandan } \\
\text { (Eng.) }\end{array}$ & $\begin{array}{l}\text { FEUH } \\
003149\end{array}$ & $\begin{array}{l}\text { Not na- } \\
\text { tive }\end{array}$ & Lf & $\begin{array}{l}\text { Used as in- } \\
\text { gredients } \\
\text { for cook- } \\
\text { ing }\end{array}$ & Food & 0.06 \\
\hline 54 & $\begin{array}{l}\text { Phyl- } \\
\text { lanthaceae }\end{array}$ & $\begin{array}{l}\text { Phyl- } \\
\text { lanthus ni- } \\
\text { ruri L. }\end{array}$ & $\begin{array}{l}\text { sampa-sam- } \\
\text { palukan } \\
\text { (Tag.) }\end{array}$ & $\begin{array}{l}\text { FEUH } \\
003181\end{array}$ & $\begin{array}{l}\text { Not na- } \\
\text { tive }\end{array}$ & Lf & $\begin{array}{l}\text { Apply } \\
\text { young } \\
\text { leaves on a } \\
\text { forehead }\end{array}$ & $\begin{array}{l}\text { Head } \\
\text { Ache }\end{array}$ & 0.09 \\
\hline 55 & Piperaceae & $\begin{array}{l}\text { Piper betle } \\
\text { L. }\end{array}$ & $\begin{array}{l}\text { gawed } \\
\text { (Dgt., Ilk,. } \\
\text { Png.) }\end{array}$ & $\begin{array}{l}\text { FEUH } \\
003176\end{array}$ & Native & Lf & $\begin{array}{l}\text { Drink de- } \\
\text { coction }\end{array}$ & Cough & 0.03 \\
\hline 56 & $\begin{array}{l}\text { Plantagina- } \\
\text { ceae }\end{array}$ & $\begin{array}{l}\text { Scoparia } \\
\text { dulcis L. }\end{array}$ & $\begin{array}{l}\text { par- } \\
\text { paraangan } \\
\text { (Ilk.) }\end{array}$ & $\begin{array}{l}\text { FEUH } \\
003170\end{array}$ & $\begin{array}{l}\text { Not na- } \\
\text { tive }\end{array}$ & Lf & $\begin{array}{l}\text { Drink de- } \\
\text { coction }\end{array}$ & $\begin{array}{l}\text { Stomach } \\
\text { Ache }\end{array}$ & 0.03 \\
\hline 57 & Poaceae & $\begin{array}{l}\text { Eleusine } \\
\text { indica (L.) } \\
\text { Gaertn. }\end{array}$ & $\begin{array}{l}\text { antibiotic } \\
\text { grass (Ilk.) }\end{array}$ & $\begin{array}{l}\text { FEUH } \\
003138\end{array}$ & Native & Lf & $\begin{array}{l}\text { Apply ex- } \\
\text { tract on the } \\
\text { wound }\end{array}$ & Wounds & 0.03 \\
\hline 58 & Poaceae & $\begin{array}{l}\text { Cymbopo- } \\
\text { gon citrat- } \\
\text { us (DC.) } \\
\text { Stapf }\end{array}$ & $\begin{array}{l}\text { tanglad } \\
\text { (Bik., Bis., } \\
\text { Mbo, Tag.) }\end{array}$ & $\begin{array}{l}\text { FEUH } \\
003144\end{array}$ & $\begin{array}{l}\text { Not na- } \\
\text { tive }\end{array}$ & Lf & $\begin{array}{l}\text { Apply } \\
\text { leaves on } \\
\text { stomach }\end{array}$ & $\begin{array}{l}\text { Stomach } \\
\text { Ache }\end{array}$ & 0.03 \\
\hline 59 & Poaceae & $\begin{array}{l}\text { Maca- } \\
\text { ranga } \\
\text { tanarius } \\
\text { (L.) } \\
\text { Müll.Arg. }\end{array}$ & samak (Ilk.) & $\begin{array}{l}\text { FEUH } \\
003166\end{array}$ & Native & $\mathrm{Fr}$ & $\begin{array}{l}\text { Used for } \\
\text { liquor } \\
\text { making }\end{array}$ & Food & 0.03 \\
\hline 60 & Rubiaceae & $\begin{array}{l}\text { Morinda } \\
\text { citrifolia L. }\end{array}$ & apatot (Ilk.) & $\begin{array}{l}\text { FEUH } \\
003159\end{array}$ & Native & Lf & $\begin{array}{l}\text { Drink de- } \\
\text { coction }\end{array}$ & $\begin{array}{l}\text { Stomach } \\
\text { Ache and } \\
\text { Gastritis }\end{array}$ & 0.03 \\
\hline 61 & Rubiaceae & $\begin{array}{l}\text { Guettarda } \\
\text { speciosa L. }\end{array}$ & None & $\begin{array}{l}\text { FEUH } \\
003165\end{array}$ & Native & Lf & $\begin{array}{l}\text { Drink de- } \\
\text { coction }\end{array}$ & $\begin{array}{l}\text { Epilepsy, } \\
\text { Diarrhea, } \\
\text { and } \\
\text { Wounds }\end{array}$ & 0.03 \\
\hline 62 & Rutaceae & Citrus sp. & $\begin{array}{l}\text { gagabutin } \\
\text { (Ilk.) }\end{array}$ & $\begin{array}{l}\text { FEUH } \\
003147\end{array}$ & $\mathrm{n} / \mathrm{a}$ & Lf & Smell & $\begin{array}{l}\text { Head } \\
\text { Ache }\end{array}$ & 0.03 \\
\hline 63 & $\begin{array}{l}\text { Sapin- } \\
\text { daceae }\end{array}$ & $\begin{array}{l}\text { Pometia } \\
\text { pinnata }\end{array}$ & $\begin{array}{l}\text { sida-i (Ilk, } \\
\text { Ibg, Ivt.) }\end{array}$ & $\begin{array}{l}\text { FEUH } \\
003123 \\
\end{array}$ & Native & Fr & $\begin{array}{l}\text { Eat } \\
\text { chewed }\end{array}$ & Food & 0.03 \\
\hline
\end{tabular}




\begin{tabular}{|c|c|c|c|c|c|c|c|c|c|}
\hline No. & Family & Species & $\begin{array}{l}\text { Common } \\
\text { name }\end{array}$ & $\begin{array}{l}\text { Acces- } \\
\text { sion no. } \\
\text { of the } \\
\text { voucher } \\
\text { speci- } \\
\text { men }\end{array}$ & $\begin{array}{l}\text { Distri- } \\
\text { bution } \\
\text { in the } \\
\text { Philip- } \\
\text { pines }\end{array}$ & $\begin{array}{c}\text { Plan } \\
t \\
\text { part } \\
\text { used } \\
\text { a }\end{array}$ & $\begin{array}{l}\text { Admin- } \\
\text { istration of } \\
\text { prepara- } \\
\text { tion }\end{array}$ & $\begin{array}{l}\text { Diseases } \\
\text { or pur- } \\
\text { pose }\end{array}$ & $\begin{array}{c}\text { Use } \\
\text { value }\end{array}$ \\
\hline 64 & $\begin{array}{l}\text { Sapin- } \\
\text { daceae }\end{array}$ & $\begin{array}{l}\text { J.R.Forst. } \\
\text { \& G.Forst. } \\
\text { Le- } \\
\text { pisanthes } \\
\text { fruticosa } \\
\text { (Roxb.) }\end{array}$ & $\begin{array}{l}\text { dirig (Ibg, } \\
\text { Ilk) }\end{array}$ & $\begin{array}{l}\text { FEUH } \\
003129\end{array}$ & Native & Fr & $\begin{array}{l}\text { Eat } \\
\text { chewed }\end{array}$ & Food & 0.03 \\
\hline 65 & Sapotaceae & $\begin{array}{l}\text { Leenh. } \\
\text { Chryso- } \\
\text { phyllum } \\
\text { cainito L }\end{array}$ & $\begin{array}{l}\text { kaimito } \\
\text { (Ibg, Tag.) }\end{array}$ & $\begin{array}{l}\text { FEUH } \\
003169\end{array}$ & $\begin{array}{l}\text { Not na- } \\
\text { tive }\end{array}$ & $\begin{array}{l}\text { Fr, } \\
\text { Lf }\end{array}$ & $\begin{array}{l}\text { Apply on } \\
\text { forehead; } \\
\text { Eat } \\
\text { chewed }\end{array}$ & Fever & 0.03 \\
\hline 66 & Urticaceae & $\begin{array}{l}\text { Pipturus } \\
\text { asper } \\
\text { Wedd. }\end{array}$ & $\begin{array}{l}\text { takop takop } \\
\text { (Ilk.) }\end{array}$ & $\begin{array}{l}\text { FEUH } \\
003120\end{array}$ & Native & Lf & - & $\begin{array}{l}\text { Handi- } \\
\text { craft }\end{array}$ & 0.03 \\
\hline 67 & $\begin{array}{l}\text { Zingibera- } \\
\text { ceae }\end{array}$ & $\begin{array}{l}\text { Zingiber } \\
\text { officinale } \\
\text { Rosc. }\end{array}$ & $\begin{array}{l}\text { pulang luya } \\
\text { (ManyLgs.) }\end{array}$ & $\begin{array}{l}\text { FEUH } \\
003140\end{array}$ & $\begin{array}{l}\text { Not na- } \\
\text { tive }\end{array}$ & $\mathrm{Fr}$ & $\begin{array}{l}\text { Apply the } \\
\text { extracts to } \\
\text { affected } \\
\text { parts }\end{array}$ & Arthritis & 0.03 \\
\hline 68 & $\begin{array}{l}\text { Zingibera- } \\
\text { ceae }\end{array}$ & $\begin{array}{l}\text { Curcuma } \\
\text { longa L. }\end{array}$ & $\begin{array}{l}\text { dilaw na } \\
\text { luya } \\
\text { (ManyLgs.) }\end{array}$ & $\begin{array}{l}\text { FEUH } \\
003141\end{array}$ & $\begin{array}{l}\text { Not na- } \\
\text { tive }\end{array}$ & $\mathrm{Fr}$ & $\begin{array}{l}\text { Drink de- } \\
\text { coction }\end{array}$ & Cancer & 0.03 \\
\hline 69 & $\begin{array}{l}\text { Zingibera- } \\
\text { ceae }\end{array}$ & $\begin{array}{l}\text { Alpinia fla- } \\
\text { bellata } \\
\text { Ridl. }\end{array}$ & banai (Ilk.) & $\begin{array}{l}\text { FEUH } \\
003172\end{array}$ & Native & Lf & $\begin{array}{l}\text { Drink de- } \\
\text { coction }\end{array}$ & $\begin{array}{l}\text { Muscle } \\
\text { pain }\end{array}$ & 0.03 \\
\hline 70 & $\begin{array}{l}\text { Zingibera- } \\
\text { ceae }\end{array}$ & $\begin{array}{l}\text { Kaempfe- } \\
\text { ria ga- } \\
\text { langa L. }\end{array}$ & disol (Ilk.) & $\begin{array}{l}\text { FEUH } \\
003155\end{array}$ & $\begin{array}{l}\text { Not na- } \\
\text { tive }\end{array}$ & Lf & $\begin{array}{l}\text { Apply ex- } \\
\text { tract on the } \\
\text { wound }\end{array}$ & $\begin{array}{l}\text { Infec- } \\
\text { tious Dis- } \\
\text { eases }\end{array}$ & 0.03 \\
\hline
\end{tabular}

Tabel 3. Informant Consensus Factor (FIC) values of the ailments or diseases cured by the medicinal plants. Abbreviations: NUR: number of use report; NT: number of plant taxa; FL: Fidelity Level

\begin{tabular}{llcccc}
\hline \multicolumn{1}{c}{ Disease Categories } & Reported diseases or uses under each UC & NUR & NT & FIC & FL (\%) \\
\hline Respiratory Diseases & Cough, Cold & 11 & 7 & 0.4 & 100.00 \\
Open Wounds & Wound, Swollen wound & 7 & 7 & 0 & 58.33 \\
Nervous System Diseases & Headache, Fever & 6 & 6 & 0 & 75.00 \\
Digestive Diseases & Stomachache, Tooth sensitivity & 4 & 4 & 0 & 44.44 \\
Parasitic Diseases & Malaria & 4 & 4 & 0 & 28.57 \\
Endocrine Diseases & Diabetes & 2 & 2 & 0 & 50.00 \\
Genitourinary Diseases & Kidney, Urinary Tract Infection & 6 & 5 & 0.2 & 100.00 \\
Postpartum Period & Menstrual Pain & 4 & 4 & 0 & 100.00 \\
Subcutaneous Tissue & Boil & 1 & 1 & 0 & 100.00 \\
Musculoskeletal Diseases & Spasm, Sprain, Arthritis & 5 & 4 & 0.25 & 66.67 \\
Cancer & Cancer & 1 & 1 & 0 & 100.00 \\
Circulatory System & Hypertension, High and Low Blood Pressure & 4 & 3 & 0.33 & 57.14 \\
\hline
\end{tabular}

Fidelity level

Medicinal plants were found to have the highest fidelity level (100\%) on the five ailment/disease categories, namely respiratory, genitourinary, postpartum period, subcutaneous tissue, and cancer diseases. However, parasitic diseases were noted to have the lowest FL with $28.57 \%$ as shown in Table 3. High FL values (near or 100\%) indicate the most preferred species for that ailment/disease. Low FL values indicate multiple purposes, so 
plants in this study were used for different purposes.

\section{Informant consensus factor}

The obtained FIC values varied from 0 to 0.40 with an average value of 0.24 . The plants were tabulated, and the top 10 categories were listed in Table 3. Respiratory diseases obtained the highest FIC value of 0.40 with 11 use reports for seven plant species. The species with the highest use report was Coleus amboinicus Lour. (oregano) with 11 use reports. The high FIC value for respiratory diseases is probably due to the humid weather condition of the island causing respiratory problems such as cough or cold. Species with high FIC value are the best candidates to search for bioactive compounds [37]. Genitourinary diseases obtained the lowest FIC value of 0.20 with six use reports for five plant species used to cure urinary tract infections (UTI). The low FIC value for the category could be due to frequent drinking of water among the locals. Good hydration by drinking water adequately will help dilute the urine and ensure urination occurs more frequently, allowing bacteria to be flushed before it reaches the bladder causing infections [38].

Other ethnobotanical uses of plants such as handicrafts and ornamentals were not included in the use categories. Monitoring studies are vital to establishing the real profile of plant species in a protected area [39]. Traditional uses of plants may change through the passage of time in terms of usability, preference, and intensity [4,18]. Hence, conservation efforts and government initiatives must be recommended to maintain plant sustainability [40]. This can be made possible with the help of community-based environmental management together with the indigenous communities in saving forest resources [41].

\section{Conclusion}

This study showed that the people of Camiguin Island, Calayan, Cagayan use 70 species of terrestrial plants belonging to 41 plant families, 35 of which are native to the Philippines. This documentation of ethnobotanical knowledge in the island will consist of the inhabitants of Camiguin Island. This study will also allow their traditional knowledge to be recognized and utilized by the scientific community, such as drug discovery, resource management, policy-making studies, conservation, and search for bioactive compounds using our native plants.

\section{Acknowledgement}

The authors are thankful to the 33 informants of our study in Camiguin Island for their active participation and support in during the interview; the Department of Environment and Natural Resources (DENR) Region II, PENRO Cagayan, CENR Sanchez Mira, and Cecile R. Luzuriaga for the facilitation and issuance of the gratuitious permit; the local government units (LGU) of Camiguin Island including Brgy. Captain Leo Batislaon of Balatubat, Brgy. Captain Anastasio Tangonan of Minabel, Brgy. Captain Gina Payas, and all their staff for their assistance during the interview; Awit Llopis for the accommodation and help during our stay in the island; Kuya Henry and Jun Jun Onate, our field guides, for their assistance during collection of samples; Erron Bacalan for the help in doing the statistical analysis; Danilo Tandang of $\mathrm{PNH}$ for the help identifying the plant specimens; and the Far Eastern University Herbarium (FEUH) for the facilities and equipment.

\section{References}

1. Schultes R, Von RS (2008) Ethnobotany. Portland, Or Timber Press.

2. Balick M, Cox P (1996) Plants, people, and culture: the science of ethnobotany. Scientific American Library.

3. Balangcod T, Balangcod A (2009). Underutilized plant resources in Tinoc, Ifugao, Cordillera Administrative Region, Luzon Island, Philippines. Acta Horticulturae 806:647-54.

4. Dapar MLG, Alejandro GJD (2020) Ethnobotanical studies on indigenous communities in the Philippines: current status, challenges, recommendations and future perspectives. Journal Complementary Medicine Research 11(1):432-46. doi: 10.5455/jcmr.2020.11.01.51

5. Jamera JKAM, Manting MME, Dapar MLG (2020) Ritual plants used by the Manobo tribe of Surigao del Sur, Philippines. Asian Journal of Ethnobiology 3(2):41-50. doi: 10.13057/asianjethnobiol/y030201

6. Dapar MLG, Demayo CG, Senarath WTPSK (2018) Antimicrobial and cellular metabolic inhibitory properties of the ethanolic extract from the bark of 'Lunas-Bagon' ( $\mathrm{Lu}$ nasia sp.). International Journal of Pharmaceutical Sciences and Research 9: 88-97. doi: 10.13040/IJPSR.09758232.9(1).88-97

7. Abdulaziz AA, Dapar MLG, Manting MME, et al. (2019) Qualitative evaluation of the antimicrobial, antioxidant, and medicinally important phytochemical constituents of the ethanolic extracts of the leaves of Gliricidia sepium (Jacq.). Pharmacophore 10: 72-83.

8. Añides JA, Dapar MLG, Aranas AT, et al. (2019). Phytochemical, antioxidant and antimicrobial properties of the white variety of 'Sibujing' (Allium ampeloprasum). Pharmacophore 10:1-12. 
9. Dela Peña JF, Dapar MLG, Aranas AT, et al. (2019). Assessment of antimicrobial, antioxidant and cytotoxic properties of the ethanolic extract from Dracontomelon dao (Blanco) Merr. \& Rolfe. Pharmacophore 10:18-29.

10. Nadayag J, Dapar MLG, Aranas AT, et al. (2019) Qualitative assessment of the antimicrobial, antioxidant, and phytochemical properties of the ethanolic extracts of the inner bark of Atuna racemosa. Pharmacophore 10:52-9.

11. Uy IA, Dapar MLG, Aranas AT, et al. (2019) Qualitative assessment of the antimicrobial, antioxidant, phytochemical properties of the ethanolic extracts of the roots of $\mathrm{Co}$ cos nucifera L. Pharmacophore 10:63-75.

12. Yao J, Weng Y, Dickey A, et al. (2015). Plants as factories for human pharmaceuticals: applications and challenges. International Journal of Molecular Science 16(12):28549-65. DOI: 10.3390/ijms161226122

13. Lewis WH (1992) Plants used medically by indigenous peoples. In: Nigg H.N., Seigler D. (eds) Phytochemical resources for medicine and agriculture. Springer, Boston, MA

14. Cheikhyous A, Mapaure I, Shapi M (2011) The use of some indigenous plants for medicinal and other purposes by local communities in Namibia with emphasis on Oshikoto Region: a review. Research Journal of Medicinal Plant 5 (4):406-19.

15. Balinado LO, Chan MA (2017) An ethnomedicinal study of plants and traditional health care practices in District 7, Cavite, Philippines. p 1-13. Presented on 2017 International Conference on Chemical, Agricultural, Biological and Medical Sciences. doi: 10.17758/uruae.ae0117622

16. Mina EC, Mina JF (2017) Ethnobotanical survey of plants commonly used for diabetes in Tarlac of Central Luzon Philippines. International Medical Journal of Malaysia 16 (1): 1-8. doi: 10.31436/imjm.v16i1.354

17. Limos GBB, Judan Cruz KG, Jacinto WR (2018) Quorum sensing inhibition bioactivities of Philippine ethnobotanicals against Pseudomonas aeruginosa. International Journal of Pure and Applied Biosciences 6 (2): 47-56. doi: 10.18782/2320-7051.6338

18. Dapar MLG, Alejandro GJD, Meve U, et al. (2020a) Quantitative ethnopharmacological documentation and molecular confirmation of medicinal plants used by the Manobo tribe of Agusan del Sur, Philippines. Journal of Ethnobiology and Ethnomedicine 16:1-60. doi: 10.1186/s13002-020-00363-7

19. Dapar MLG, Demayo CG, Meve U, et al. (2020b) Molecular confirmation, constituents and cytotoxicity evaluation of two medicinal Piper species used by the Manobo tribe of Agusan del Sur, Philippines. Phytochemistry Letters 36:24-31. doi: 10.1016/j.phytol.2020.01.017

20. Reyes M, Bennett D, Oliveros C (2018). The monitor lizards of Camiguin Island, Northern Philippines. Varanidae.org.

21. Cortez G (2015). Northern Exposure. Manila Times. http://www.manilatimes.net/northern-exposure2/222195/

22. Kennedy RS, Gonzales PC, Dickinson EC, et al. (2000). A guide to the birds of the Philippines. Oxford University Press, Oxford.

23. Heaney LR, Ruedi M (1994) A preliminary analysis of biogeography and phylogeny of Crocidura from the Philippines. Special Publication 18:357-377. Carnegie Museum of Natural History.
24. Acebes JV, Lesaca LAR (2003) Research and conservation of Humpback Whales (Megaptera Novaeangliae) and other cetacean species in the Babuyan Islands, Cagayan Province, Northern Luzon, Philippines. In: Van der Ploeg J, Masipiquena AB, Bernardo EC (editors), The Sierra Madre Mountain Range: Global Relevance, Local Realities. Cagayan Valley. Program on Environment and Development, Golden Press, Tuguegarao City. Pp 34-42.

25. Sansanelli S, Ferri M, Salinitro M, et al. (2017) Ethnobotanical survey of wild food plants traditionally collected and consumed in the Middle Agri Valley (Basilicata Region, Southern Italy). Journal of Ethnobiology and Ethnomedicine 13 (50): 1-10. doi: 10.1186/s13002-0170177-4

26. Chase MW, Hills HH (1991) Silica gel: an ideal material for preservation of leaf samples for DNA studies. Taxon 40: 215-20. doi: 10.2307/1222975

27. Pelser PB, Barcelona JF, Nickrent DL (eds) (2011 onwards) Co's Digital Flora of the Philippines. www.philippineplants.org

28. Allen P, Bennett K (2010) PASW Statistics by SPSS: A Practical Guide: Version 18.0 (1st ed.). South Melbourne, Victoria, Australia: Cengage Learning Australia. ISBN 9780170222907

29. Martin GJ (2004) Ethnobotany: a methods manual. London: Earthscan Publications. p 17.

30. Amiguet VT, Arnason JT, Maquin P, et al. (2005) A consensus ethnobotany of the Q'eqchi' Maya of southern Belize. Economic Botany 59:29-42. doi: 10.1663/00130001(2005)059[0029:ACEOTQ]2.0.CO;2

31. Dapar MLG, Meve U, Liede-Schumann S, et al. (2020) Ethnomedicinal plants used for the treatment of cuts and wounds by the Agusan Manobo of Sibagat, Agusan del Sur, Philippines. Ethnobotany Research and Applications 19(31):1-18. DOI: 10.32859/era.19.31.1-18

32. Dapar MLG, Alejandro GJD, Meve U, et al. (2020) Ethnomedicinal importance and conservation status of medicinal trees among indigenous communities in Esperanza, Agusan del Sur, Philippines. Journal of Complementary Medicine Research 11(1):59-71. doi: 10.5455/jcmr.2020.11.01.08

33. Dapar MLG, Meve U, Liede-Schumann S, et al. (2020) Ethnomedicinal appraisal and conservation status of medicinal plants among the Manobo tribe of Bayugan City, Philippines. Biodiversitas 21 (8):3843-3856. doi: 10.13057/biodiv/d210854

34. Abe R, Ohtani K (2013) An ethnobotanical study of medicinal plants and traditional therapies on Batan Island, the Philippines. Journal of Ethnopharmacology 145(2):554-65. doi: 10.1016/j.jep.2012.11.029

35. Langenberger G, Prigge V, Martin K, et al. (2009) Ethnobotanical knowledge of Philippine lowland farmers and its application in agroforestry. Agroforestry System 76: 173-194.

36. Tantengco OAG, Condes MLC, Estadilla HHT, et al. (2018) Ethnobotanical survey of medicinal plants used by Ayta communities in Dinalupihan, Bataan, Philippines. Pharmacognosy Journal 10 (5):859-70.

37. Chikezie P, Ibegbulem C, Mbagwu F (2015) Bioactive principles from medicinal plants. Research Journal of Phytochemistry 9:88-115.

38. Beyer M (2018) Drinking water lower the risk of bladder infections. https://www.medicalnewstoday.com/articles/323267.php 
39. Cudal MG, Calimbo LGL, Sumibay Jr. D, et al. (2021). Diversity of pteridophytes in Mount Timolan Protected Landscape, Zamboanga del Sur, Philippines. Journal of Tropical Life Science 11 (2): 161-70. doi: 10.11594/jtls.11.02.05

40. Nasution A, Chikmawati T, Walujo EB, et al. (2018) Ethnobotany of Mandailing Tribe in Batang Gadis National
Park. Journal of Tropical Life Science 8 (1):48-54. doi: 10.11594/jtls.08.01.09

41. Andesmora EV, Muhadiono, Hilwan I (2017) Ethnobotanical study of plants used by people in Hiang Indigenous Forest Kerinci, Jambi. Journal of Tropical Life Science 7 (2):95-101. doi: 10.11594/jtls.07.02.02

42. Madulid DA (2001) A dictionary of Philippine plant names. Makati City, Bookmark 